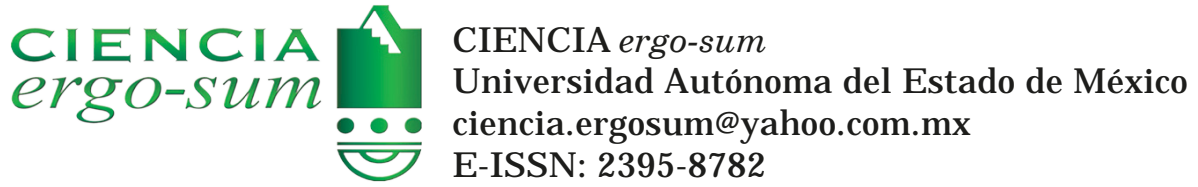

\title{
Mapa de ruta bio jet en México: construcción, análisis y puntos de reflexión
}

Mar J uárez, Elizabeth

Mapa de ruta bio jet en México: construcción, análisis y puntos de reflexión

CIENCIA ergo-sum, vol. 28, núm. 1, marzo-junio 2021 |e115

Universidad Autónoma del Estado de México, México

Esta obra está bajo una Licencia Creative Commons Atribución-NoComercial-SinDerivar 4.0 Internacional .

Mar J uárez, E. (2021). Mapa de ruta bio jet en México: construcción, análisis y puntos de reflexión. CIENCIA ergo-sum, 28(1). https://doi.org/10.30878/ces.v28n1a9 


\title{
Mapa de ruta bio jet en México: construcción, análisis y puntos de reflexión
}

\section{Bio Jet route map in Mexico: construction, analysis and reflection points}

\author{
Elizabeth Mar Juárez \\ Instituto Mexicano del Petróleo, México \\ emar@imp.mx \\ (1) http://orcid.org/0000-0001-5124-7191
}

Recepción: 28 de enero de 2020

Aprobación: 14 de julio de 2020

\begin{abstract}
RESUMEN
La necesidad de un mundo con bajas emisiones de carbono ha agregado nuevos desafíos en la planeación a largo plazo. La construcción de este mapa de ruta es proporcionar una visión de cómo podría suceder un futuro en donde se tenga un mercado nacional de Bio Jet. Elaborado desde la perspectiva nacional de la Secretaría de Energía más inteligencia tecnológica, se construye un mapa desde la conciencia y la ciencia. Se proyecta una revisión y se localizan puntos rojos donde se requiere un reanálisis de datos para generar iniciativas emergentes y proponer nuevos entornos para focalizarse en ideas y tecnologías diferentes que al final permitan desarrollar/implementar un mercado nacional de bioenergéticos.
\end{abstract}

Palabras Claves: mapa de ruta Bio Jet, biocombustibles sector aéreo.

\section{Abstract}

The need for a world with low carbon emissions has added new challenges in long-term planning. The construction of this roadmap is to provide a vision of how a future could happen in which there is a national biojet market. Prepared from the national perspective of the Ministry of Energy plus technological intelligence, to construct a map from consciousness and science, and what is rarely to do. A revision is projected and red dots are located where a new analysis of data to generate emerging initiatives and propose new environments to focus on different ideas and technologies that will eventually allow the development / implementation of a national bioenergy market.

KEYWORDS: Bio Jet route map, biofuels air sector.

\section{INTRODUCCIÓN}

La firma del Acuerdo de París y su ratificación por parte de México nos ofrece la oportunidad de reflexionar sobre lo que hemos logrado, lo que aún tenemos que hacer y cómo conseguirlo en términos más específicos. En estas metas nacionales se encuentran involucradas la ciencia, la sociedad, la política y la economía teniendo como último objetivo el construir un futuro bajo en carbono.

Así, 2008 marca la puesta en marcha de diversas políticas energéticas y climáticas locales, nacionales e internacionales que ganan gran relevancia, dando paso a una serie de eventos coincidentes:

a) Una verdad incómoda (título original An Inconvenient Truth) es un documental del director Davis Guggenheim acerca de la campaña del exvicepresidente de Estados Unidos Al Gore para educar a los ciudadanos sobre el calentamiento global. Ganó el Oscar en 2007 como mejor largometraje documental; su éxito fue tal en las salas de cine que hizo del calentamiento global un tema debatido públicamente. En una encuesta de 47 países, 66\% de los encuestados que había visto la película declaró que había cambiado de opinión sobre el calentamiento global y $89 \%$ manifestó que se hizo más consciente del problema (Nielsen-Gammon, 2007). 
b) Se publican en diversos países leyes y estrategias nacionales para prevenir, evitar y mitigar el cambio climático: Reino Unido en 2008, México en 2012 y China en 2013 se comprometieron con el Plan Estratégico para el Medio Ambiente.

c) De manera paralela se lanza un sistema de comercio de emisiones de gases de efecto invernadero (GEI) entre países y/o entre regiones (principalmente en Europa) que se anticiparon al mismo mercado de Kyoto, como es el caso del UK Emission Trading Scheme nacido en el Reino Unido en noviembre de 2000. En este caso el programa Bretón tenía como objetivo reflejar la importancia del cambio climático al buscar con este esquema reducir las emisiones de los gases de efecto invernadero en un 20\% para 2010, en comparación con los niveles de 1990, y equilibrar los esfuerzos de mitigación entre sectores y al mismo tiempo salvaguardando la competitividad. Otro esquema fue el de la Unión Europea denominado Emission Trading Scheme (ETS) que aplica en todo su territorio, y que, en el último caso, admite el comercio de unidades de carbono previstas por el protocolo.

A pesar de todos los esfuerzos mencionados, la crisis financiera y económica global desatada a finales de 2008 causó diversos fenómenos económicos que afectaron muchas de las acciones orientadas a la mitigación de emisiones como: una crisis alimentaria global, diferentes derrumbes bursátiles, cambios en apoyos y programas ambientales en el mundo desarrollado y, a medida que se profundizaron los efectos económicos de la crisis financiera, los precios de los permisos de emisiones de dióxido de carbono o de contaminar bajaron de precio hasta 12 EUR por tonelada emitida (15.58 USD/ton $\mathrm{CO}_{2}$ ), cuando se había cotizado a un precio de aproximadamente 30 EUR por tonelada (38.94 USD).

De manera particular, la crisis económica en Europa llevó a una drástica disminución en las emisiones de bonos de $\mathrm{CO}_{2}$ dentro del bloque del G-27. Como resultado, la demanda de permisos (derechos a emitir) se redujo y por consecuencia los precios han disminuido. En 2011, el precio de los derechos de emisiones del Régimen de Comercio de Emisiones de la Unión Europea (ETS) se ubicaba en unos 7 EUR por tonelada (Borghesi y Montini, 2016).

Todo esto afecto de manera directa la puesta en marcha de diversos mecanismos para reducir emisiones, entre ellos, el uso extendido de biocombustibles. Si al tener un revés económico provoco serios cambios en las metas ambientales, es momento de crear nuevos caminos o tomar los anteriores y darles un giro para que cumplan su función. Hay algo que no se puede negar: actualmente se presentan más fenómenos meteorológicos extremos (incendios forestales, inundaciones y sequías), lo que está haciendo que el cambio climático sea considerado real para muchas personas. En este sentido, surge la incorporación de productos deseables bajos en carbono en el mercado, desde el vehículo eléctrico hasta hamburguesas imposibles. Resulta curioso la forma en que una hamburguesa nace como un producto de bajo carbono al tratar de mostrar que se puede cambiar el esquema agroalimentario actual, el cual consideran sus creadores ha contribuido en gran medida al cambio climático. Empresas como Impossible Foods lideran una revolución tecnológica alimentaria, de la cual su primer producto es la Impossible Burger, comercializada ya en los EE. UU., y que está producida con tesina. Esta "hamburguesa de plantas" pretende reemplazar a la hamburguesa de ternera convencional al proporcionar una experiencia sensorial idéntica o mejorada. Pero su ingrediente estrella, la heme, marca la diferencia con el resto de hamburguesas vegetarianas; es la pieza clave que determinará el futuro comercial de este producto en Europa. De este modo, se tienen nuevos productos que tratan de modificar los mercados tal como se conocen o se incorporan productos "amables" con el ambiente mostrando a la gente que existe una alternativa positiva.

De acuerdo con los movimientos de ciudadanos respecto al cambio climático, la percepción es que la respuesta sucede a un ritmo lento, pero también incide en los mercados. Por ejemplo, se tiene el movimiento civil de Greta Thunberg: la School strike for climal and Extinction Rebellion. Esta activista climática sueca de 16 años creó el movimiento Huelga Escolar por el Clima, para contar la historia de este momento: “iNuestra casa está 
en llamas! No quiero tu esperanza. Quiero que sientas el miedo. Quiero que actúes como si la casa estuviera en llamas. Porque así es" (Thunberg, 2019). Desde agosto de 2018, cuando Greta decidió violar la ley sueca e ir a la huelga al sentarse frente al Parlamento de Suecia, se sumaron 270 escuelas de diversas partes del mundo, desde Australia, Japón y hasta Uganda, con cientos de miles de escolares que tomaban las calles para demandar acciones y con movimientos programados en 2019 hasta que se escuchen sus demandas.

En el caso del reciente Informe Especial sobre el Calentamiento Global de $1.5^{\circ} \mathrm{C}$ del IPCC (Intergovernmental Panel on Climate Change) fue una lectura aleccionadora para muchos políticos y líderes empresariales que pensaron que el tiempo estaba de nuestra parte, donde todavía se tenía un periodo suficiente para dejar las cosas y que sus sucesores las resolvieran. En este informe del IPCC se informa sobre los impactos del calentamiento global de $1.5^{\circ} \mathrm{C}$ por encima de los niveles preindustriales y las vías mundiales relacionadas con las emisiones de gases de efecto invernadero, en el contexto del fortalecimiento de la respuesta mundial a la amenaza del cambio climático, la búsqueda del desarrollo sostenible y los esfuerzos para erradicar la pobreza. Uno de los mensajes clave de este informe es que ya son notorias las consecuencias del calentamiento global a $1{ }^{\circ} \mathrm{C}$ visible en un clima más extremo, el aumento del nivel del mar y la disminución del hielo marino en el Ártico, entre otros cambios. El informe destaca una serie de impactos del cambio climático que podrían evitarse si se limitara el calentamiento global a $1.5^{\circ} \mathrm{C}$ en comparación con $2^{\circ} \mathrm{C}$ o más. Por ejemplo, para 2100 , el aumento del nivel del mar global sería $10 \mathrm{~cm}$ más bajo con un calentamiento global de $1.5^{\circ} \mathrm{C}$ en comparación con uno de $2^{\circ} \mathrm{C}$. La probabilidad de un océano Ártico libre de hielo marino en verano sería una vez por siglo con un calentamiento global de $1.5^{\circ} \mathrm{C}$, comparado con al menos una vez por década con $2{ }^{\circ} \mathrm{C}$. Los arrecifes de coral disminuirían en un $70-90 \%$ con un calentamiento global de $1.5^{\circ} \mathrm{C}$, mientras que prácticamente todos (>99\%) se perderían con $2{ }^{\circ} \mathrm{C}$.

Por ello, uno de los mensajes fundamentales presentados por el Grupo Intergubernamental de Expertos sobre el Cambio Climático (IPCC por sus siglas en inglés) es crear un sentido de urgencia para el desarrollo sostenible debido a que ya se viven las consecuencias de un calentamiento global de $1{ }^{\circ} \mathrm{C}$ con condiciones meteorológicas más extremas, crecientes niveles del mar y un menguante hielo marino en el Ártico, entre otros muchos cambios globales (IPCC, 2018).

En consecuencia, el informe del IPCC expone como solución limitar el calentamiento global a $1.5^{\circ} \mathrm{C}$. Para alcanzar esta meta se requerirán transiciones "rápidas y de gran alcance” en los diferentes ámbitos económicos, de la energía, de la industria, del sector servicios, en el transporte y en las ciudades. De acuerdo con el IPCC, las emisiones netas globales de dióxido de carbono $\left(\mathrm{CO}_{2}\right)$ causadas por los seres humanos tendrían que disminuir en aproximadamente un 45\% respecto a los niveles de 2010 en 2030, y alcanzarían el cero neto alrededor de 2050. Esto significa que cualquier emisión restante debería equilibrarse eliminando ese $\mathrm{CO}_{2}$ del aire.

Las apuestas por alcanzar rápidamente la neutralidad en emisiones de carbono a nivel global se centran en acciones tendientes a la generación de electricidad verde en el corto plazo, es decir, aumentar de manera gradual la proporción de energía renovable que se genera y se compra a partir de fuentes renovables como la solar y la eólica. Es así como la electrificación es vista como una estrategia prometedora para descarbonizar diversos sectores. Sin embargo, este tipo de medida no aplica para todos los sectores. Por ejemplo, en el caso del sector transporte y, en específico, del transporte aéreo las oportunidades son más limitadas en términos de electrificar aeronaves; este modo de transporte enfrenta altas barreras a la electrificación debido a los requisitos de seguridad, sensibilidad al peso y demandas de alto rendimiento.

En este caso, la introducción de un consumo extendido de electricidad en sustitución de combustibles líquidos requerirá que se realicen avances sustanciales en diversos campos tecnológicos, por lo que la posibilidad de emplear tecnologías de emisión cero dentro de la aviación con referente a la electrificación probablemente permanecerá en aplicaciones específicas como taxis aéreos y aviones de pasajeros de corta distancia. Todavía es más probable que el sector de la aviación siga dependiendo de los combustibles líquidos hasta 2050, en especial para vuelos de larga distancia. 
De acuerdo con Hall et al. (2018), el potencial de las tecnologías de cero emisiones para reducirlas en el sector transporte están llegando a diferentes ritmos de progreso, pero continuando con la idea de descarbonizar los diferentes sectores y apropiarse de las metas del IPCC que dicen que "es posible limitar el calentamiento a 1.5 ${ }^{\circ} \mathrm{C}$ dentro de las leyes de química y física, pero para hacerlo se requerirán cambios sin precedentes” (Jim Skea, Copresidente del Grupo de trabajo III del IPCC, 2018). En el análisis de este artículo se construye un escenario para el sector aéreo mexicano con el objetivo de reducir los gases de efecto invernadero (GEI) generados por sus operaciones en contraparte con un escenario donde no cambia la tendencia de consumo. Para lograr estas reducciones se realiza una sustitución de combustibles con baja huella de carbono (Bio Jet) y de igual manera se realiza una valoración técnico-económica del costo que implicaría la introducción de estos combustibles en la matriz energética nacional.

En México desde 2006 con la Estrategia Nacional de Cambio climático se ha tenido un recorrido de más de 12 años en la búsqueda de acciones que lleven a un México con bajas emisiones de carbono. Ahora es el momento de reflexionar sobre lo que funcionó, lo que no ha funcionado y cómo se abordará todo lo que aún queda por hacer (figura 1).

El análisis de este artículo es una serie de tres documentos basados en la introducción de biomasa/bioenergético en el sector transporte mexicano; en este apartado en especial se aborda la temática del sector aéreo y los compromisos internacionales que tiene, los cuales lo impulsan a buscar opciones y estrategias para cumplir con las metas planteadas.

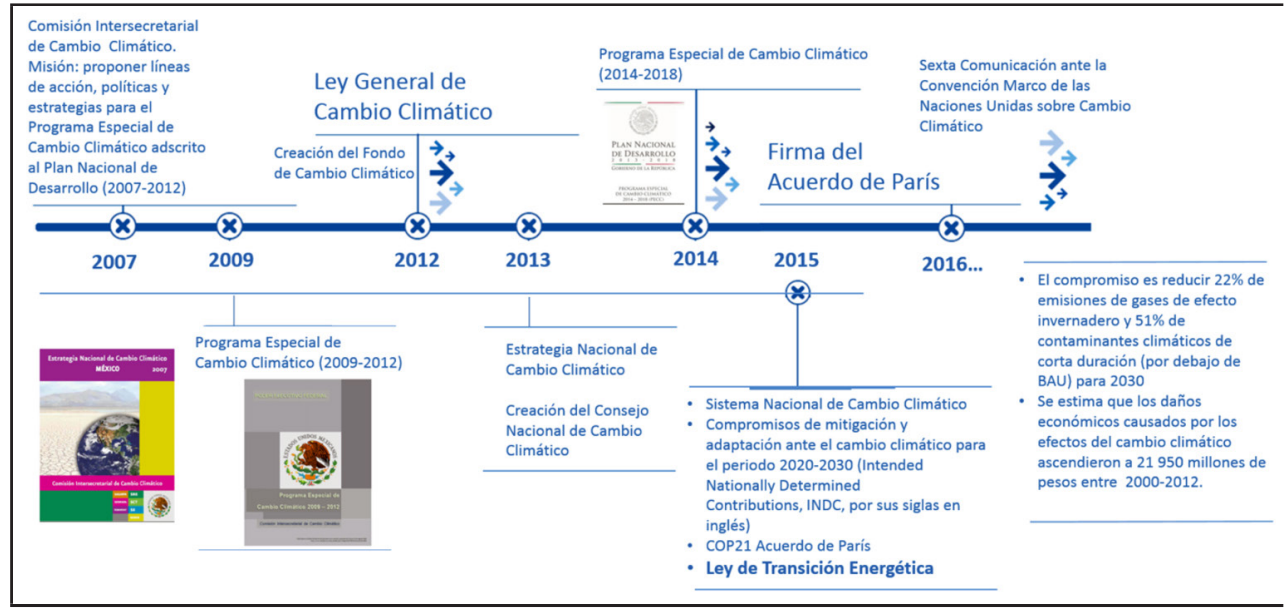

FIGURA 1

Evolución del marco oficial en México ante el cambio climático Fuente: elaboración propia.

\section{La NeCESIDAD de COMbustibles RenOvables Para la aViación}

El transporte aéreo es uno de los sectores de más rápido crecimiento. Se calcula que la demanda mundial de pasajeros por tráfico aéreo aumentó un 7.4\% en 2018 respecto al año anterior y se proyecta que el tráfico crecerá un $5 \%$ para 2019. Así, se observa que la aviación está creciendo, además de aumentar su demanda de actividad y de combustibles.

Las proyecciones a 2031 muestran un aumento en la flota de aviones en 28200 unidades nuevas tanto para pasajeros como para carga, y se estima que en 2036 viajarán 7800 millones de personas, casi el doble respecto a los 4000 millones que viajaron en 2017 de acuerdo con la última previsión de pasajeros realizada por la Asociación de Transporte Aéreo Internacional (IATA, 2017), que sitúa la tasa de crecimiento anual acumulativo en un 3.6\% subrayando con esto que las perspectivas de crecimiento a largo plazo para la industria son un hecho (figura 2). 


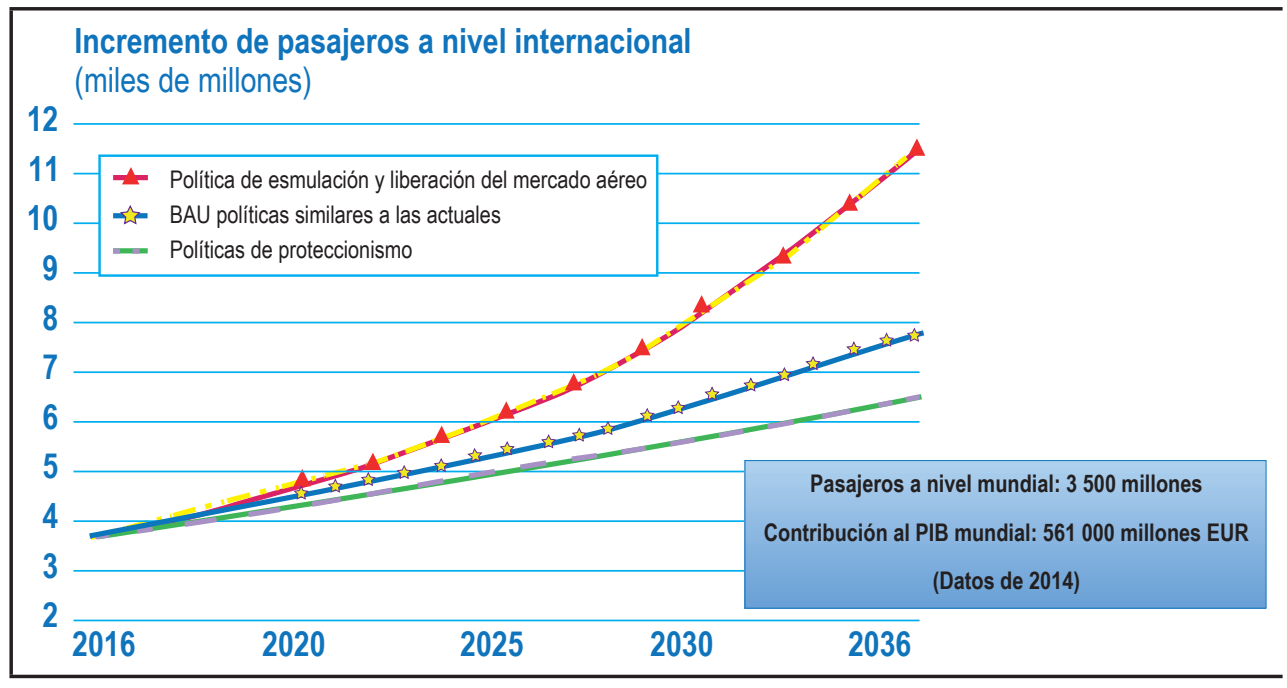

FIGURA 2

Incremento esperado de pasajeros/actividad en el sector aéreo

Sin lugar a dudas, el crecimiento esperado de la aviación es alto, por lo que se debe tener en cuenta que el transporte aéreo sólo puede ser sostenible si no compromete sus operaciones con grandes impactos en el medioambiente. Lo anterior se tuvo presente durante la COP21 en París cuando se acordó de manera internacional por diversos gobiernos limitar el aumento de la temperatura global a un máximo de $2{ }^{\circ} \mathrm{C}$. La aviación internacional aunque no se encontraba obligada por el Acuerdo de París durante la COP21, el sector aéreo se propuso limitar sus emisiones de $\mathrm{CO}_{2}$ a partir de 2020 y reducir a la mitad las emisiones de $\mathrm{CO}_{2}$ para 2050 en comparación con la línea de base de 2005. En concordancia con estas acciones, la Organización de Aviación Civil Internacional acordó recientemente introducir una medida basada en el mercado global, la cual propone compensar cualquier aumento anual en las emisiones de $\mathrm{CO}_{2}$ de la aviación internacional más allá de 2020 a través de un Esquema de Reducción y Compensación de Carbono para la Aviación Internacional (en inglés Carbon Offsetting and Reduction Scheme for International Aviation, CORSIA) (figura 3).

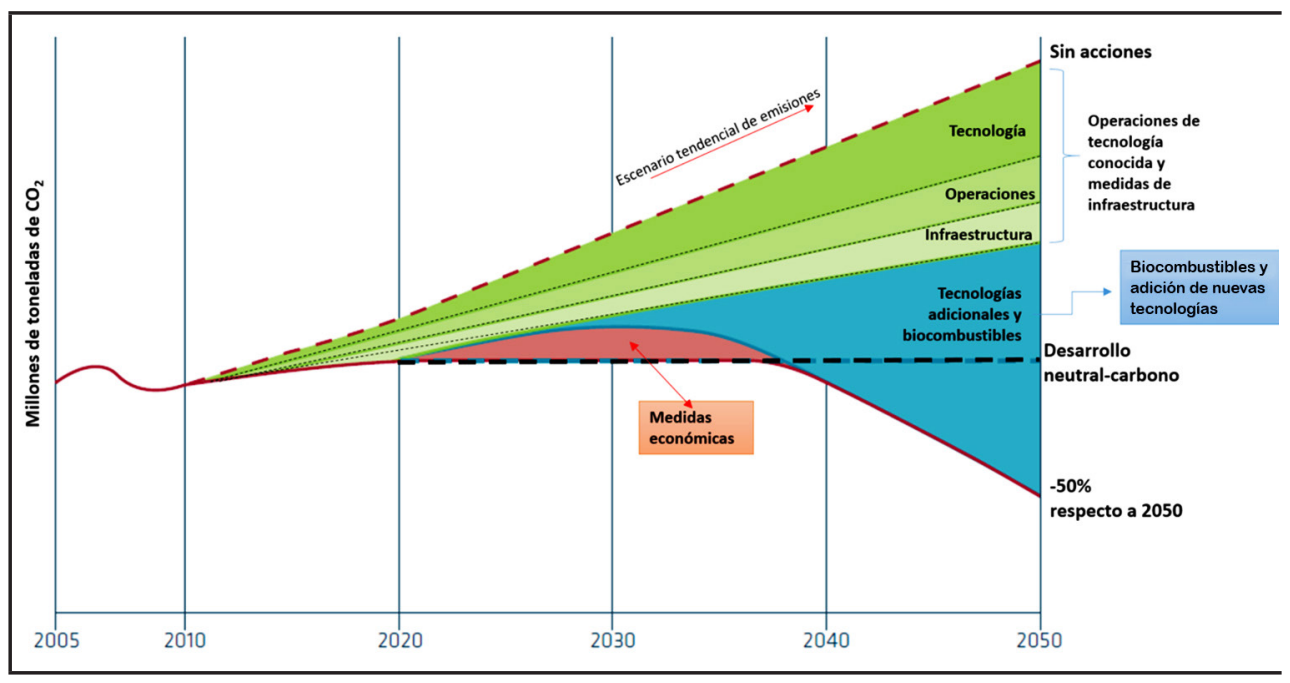

FIGURA 3

Reducción de emisiones en la industria Aérea Fuente: adaptada de la publicación en IATA Technology Roadmap 2015 (IATA, 2013). 
Otra medida para reducir los impactos de la industria aérea se basa en la eficiencia. Por esta razón, en 2010, la OACI y sus Estados miembros adoptaron ambiciosos objetivos aspiracionales para lograr una mejora mínima de $2 \%$ en eficiencia del uso del combustible, así como la introducción de combustibles alternativos a la turbosina que tengan una menor huella de carbono; de esta forma es como se plantea la introducción del Jet Fuel renovable (JFR) que se explora en este trabajo.

\section{Desarkollo de un mapa de ruta estratégica de energía para el uso de Bio Jet en méXico}

El objetivo principal de este esfuerzo es desarrollar un mapa de ruta que detallara el camino a seguir para continuar con los esfuerzos de incluir en la matriz energética de México el uso de bioenergéticos, especialmente el Bio Jet. Resulta interesante que al incluir el término bio muchos de los problemas observados en otros países se magnifican; factores como tierra disponible, transgénicos, integración agro/industria, compra de tecnología llave en mano, entre otros temas, se vuelven de mayor importancia en un entorno donde la historia sobre el uso y explotación de la tierra tiene un peso enorme en las decisiones de país. Además de las barreras sociales y ambientales, también como país se debe definir la posición de innovador, desarrollador o comprador de tecnologías que puede reducir el potencial cuando no se tienen la certeza del cambio tecnológico.

Los resultados presentados no identifican ni cuantifican todos los impactos de un mercado de Bio Jet. Reúne diversos puntos de vista, se apoya en otros mapas de rutas de Bio Jet o de algún otros bio energético que se ha publicado a lo largo de los años (Mar, 2019). La investigación proporciona un marco dentro del cual se puedan comenzar a tomar decisiones, definir una visión de lo que podría ser el futuro, y una guía para determinar qué preguntas deben seguir después de este primer paso.

\section{Metodología}

Con la finalidad de responder el cómo se incluirá la producción de Bio Jet en México, se requiere definir desde dónde se parte. Para ello, se tomará 2018 como marco de referencia con un análisis del estado de desarrollo de las tecnologías asociadas con los procesos empleados para producir bioturbosina (incluyendo las características de la materia prima empleada). Definido el punto de partida nacional, ampliamos la visión a un marco internacional sobre qué están haciendo otros mercados para establecer retos que han tenido, cómo los han solventado, las oportunidades que vislumbran en un futuro y para cuándo se cree que las alcanzarán. Cabe señalar que el contenido que se presenta en este trabajo no comprende un inventario exhaustivo de las capacidades técnicas con las que cuenta México para la producción de bioturbosina, sino información base para establecer el enfoque y alcance del mapa de ruta tecnológica.

Este esfuerzo comenzó al estudiar las tendencias, proyecciones energéticas y de tecnología de los mercados energéticos. La investigación involucró una serie de encuesta relacionadas con impactos, pasos a seguir, impulsores, barreras actuales y futuras para un mercado primero del uso de bioenergéticos y luego específicamente de Bio Jet. También se preguntó que podría alterar las tendencias pasadas hacia el futuro. Estas tres tareas ayudaron a la construcción del mapa de ruta.

Se estableció como meta evaluar el potencial de diversas tecnologías energéticas para la producción de Bio Jet y una evaluación básica del potencial de materias primas para cuantificar la viabilidad relativa de alternativas específicas para la producción de Bio Jet con base en las estadísticas de producción agrícola nacional, así como datos históricos sobre cómo se han comportado ciertos cultivos que han tratado de introducirse en el mercado nacional como la jatropha y la salicornia, de los cuales se registró tasa de éxito, producción por hectárea y expectativas de crecimiento con base en su análisis histórico. Posteriormente, se construyó un modelo simple de hoja de cálculo para evaluar el impacto potencial de las iniciativas propuestas y de las 
tecnologías de producción e identificar dónde se presentan problemas y dónde expandirse o desarrollar nuevas iniciativas que podrían ayudar a alcanzar el objetivo nacional de reducción en términos de emisiones de gases de efecto invernadero.

\section{1. Estimación del marco para el desarrollo del mapa de ruta (punto de partida: 2018)}

Estado actual: la bioturbosina o Bio Jet en México se tiene en el radar desde hace 10 años. Su introducción oficial se establece en la Ley de Promoción y Desarrollo de los Bioenergéticos (LPDB, 2008), la cual tiene como objetivo la inclusión de los bioenergéticos en la matriz energética del país. Esta ley justifica una serie de iniciativas orientadas a generar materia prima para la producción de biocombustibles, proveer herramientas para promover proyectos con energías renovables y dar apoyos para la investigación. En el ámbito internacional, IATA (2009) declara que buscará el crecimiento neutro de carbono en 2020 con una reducción a la mitad de las emisiones en 2050 en relación con las emisiones del 2005. Y así comienza el camino para la introducción de los bioenergéticos en el sector aéreo.

En noviembre de 2009, en la primera Conferencia sobre Aviación y Combustibles Alternativos de la ICAO, se estableció el marco para generar una plataforma para compartir información sobre mejores prácticas e iniciativas que faciliten el desarrollo y el despliegue de combustibles alternativos sostenibles para la aviación a nivel mundial. Muchos ensayos en vuelo y certificación de combustible han demostrado que los combustibles alternativos son una solución técnicamente viable.

En el caso nacional, se han creado las condiciones para integrar la cadena de valor que fomente un mercado de bioenergéticos en el sector aéreo. De acuerdo con ASA, en 2014 vendieron 109693 litros de bioturbosina en aeropuertos nacionales y se han promovido acciones para la implantación de biorrefinerías y producción de materia prima ex profeso para este fin. Sin embargo, no se dice si la materia prima con la que se elaboró esta bioturbosina es nacional o cuál es su procedencia, ni dónde se produjo ni se proporciona ninguna otra información. Respecto a las tecnologías en el Primer Congreso Nacional de Bioturbosina celebrado en México en 2018, se menciona que en territorio nacional se encuentra en construcción una biorrefinería en Mazatlán, Sinaloa, y otro proyecto en Tijuana, Baja California. También se encuentran datos de que la Secretaría de Energía inauguró la Planta Piloto de Microalgas en el estado de Tabasco en 2018, la cual se estima que producirá biocombustibles a partir de microalgas y costó 79 millones de pesos. En la página oficial del clúster bioturbosina se menciona que enfoca sus actividades de investigación en los procesos de hidrotratamiento de ésteres y ácidos grasos (HEFA) y Alcohol-to-Jet (ATJ) y durante el desarrollo del Congreso se menciona que también se está trabajando en pirolisis.

En resumen, se han probado diversas materias primas y se menciona el potencial, pero no se hace mención que ya se estén trabajando con el fin explícito de producción de Bio Jet; es importante mencionar que estos cultivos se pueden emplear en la producción de biodiésel y etanol, por lo que un análisis de competitividad entre combustibles bio permitiría definir el mercado hacia donde se orienta la materia prima. Respecto a la tecnología se reconocen sólo tres en proceso de investigación que son HEFA, ATJ y pirolisis, no en escala comercial. Teniendo presente lo anterior, se evalúa el mercado de bioturbosina nacional como incipiente (figura 4).

Si bien llegar a un punto donde se establece que el mercado nacional de Bio Jet es incipiente, podría generar cierta controversia si se considera que desde 2011 se han realizado vuelos empleando Bio Jet, como se muestra en la figura 5. Sin embargo, el registro de vuelos fue en 2011 (hace más de siete años); asimismo, se tiene el registro de un acuerdo en 2016 entre Aeropuertos y Servicios Auxiliares (ASA), Aeroméxico, el Instituto Potosino de Investigación Científica y Tecnológica (IPICYT) y Boeing, apoyados por el fondo sectorial del Consejo Nacional de Ciencia y Tecnología (Conacyt) y la Secretaría de Energía (Sener) con el fin de investigar y desarrollar Bio Jet sostenible en México. Pero en 2018, durante la presentación del Reporte de Sostenibilidad 2017, Sergio 
Allard, director ejecutivo de Relaciones Institucionales de Aeroméxico declara que entre las iniciativas futuras para una operación más amigable con el medioambiente, la empresa aérea contempla la posibilidad de usar combustibles con mayor eficiencia energética, entre ellos los biocombustibles. La palabra operativa es posibilidad, no es todavía una realidad. Entonces, referirnos al mercado nacional como incipiente resulta práctico.

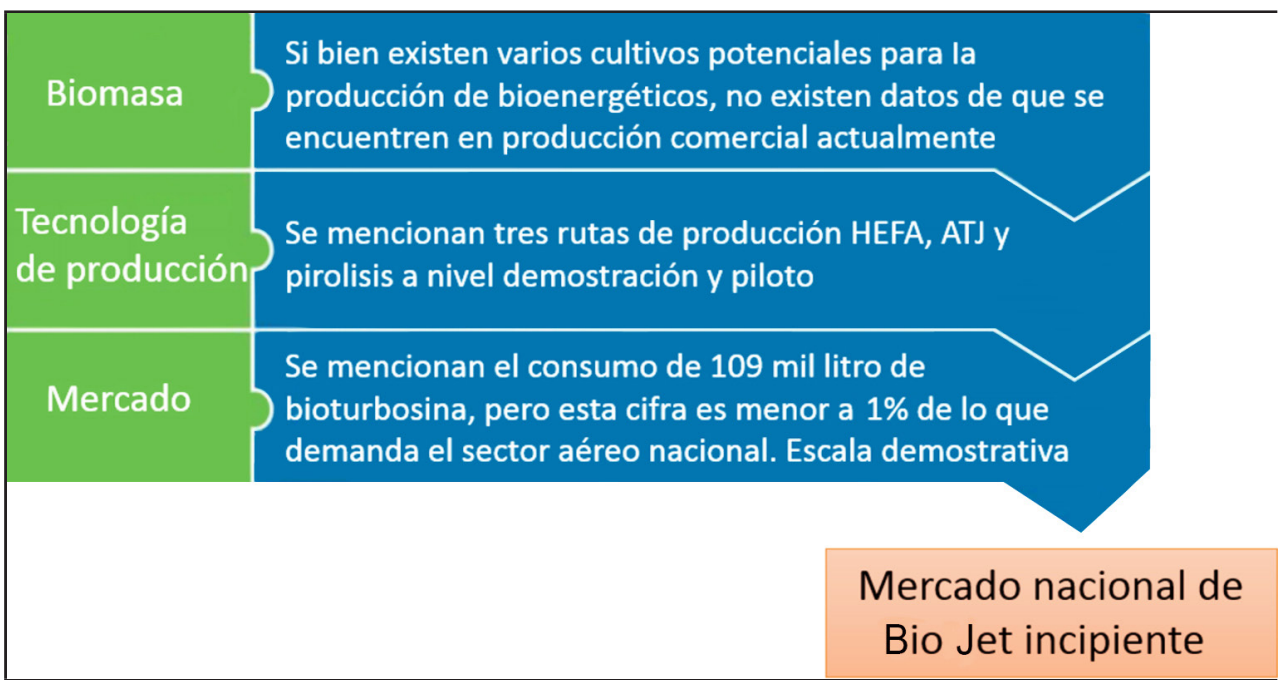

FIGURA 4

Nivel de desarrollo del mercado nacional de Bio Jet Fuente: Mar, 2019.

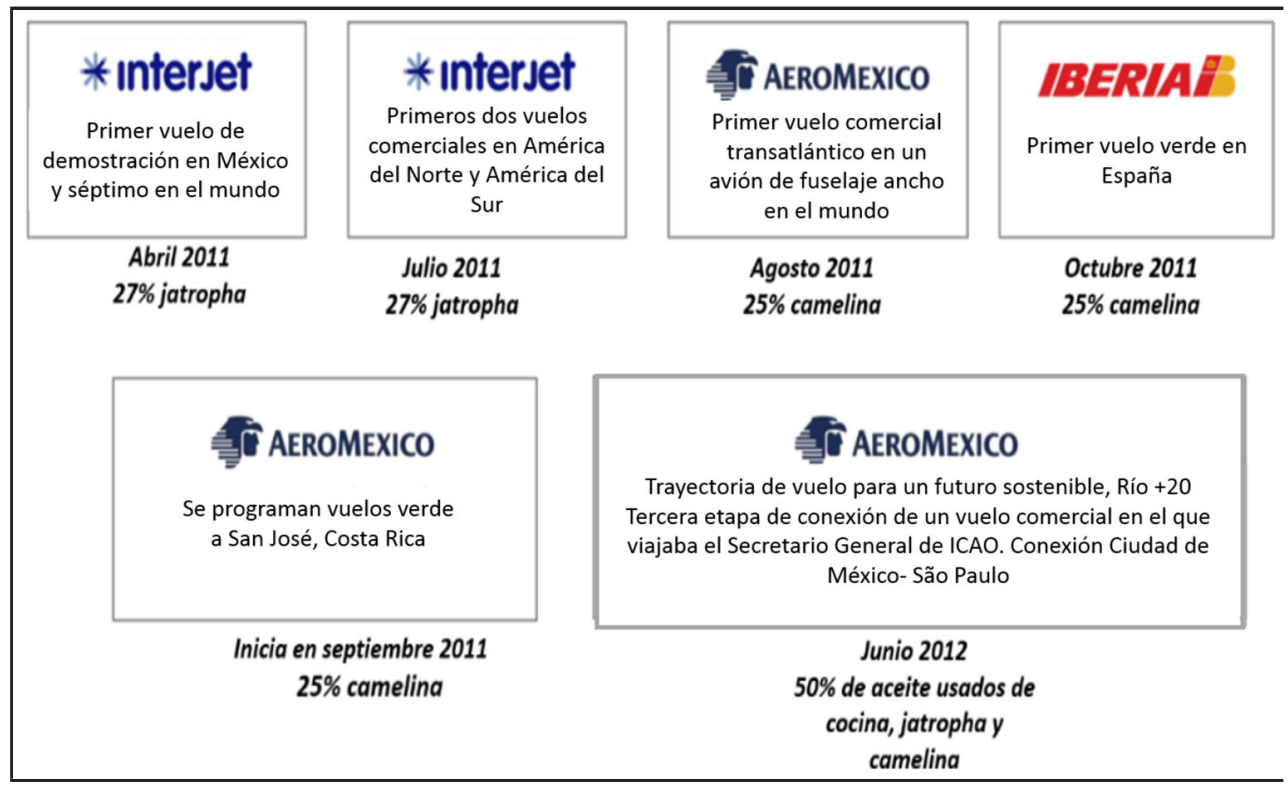

FIGURA 5

Vuelos mexicanos realizados con Bio Jet

Fuente: ASA, 2018.

\section{2. Impulsores y barreras a la entrada}

La mayoría de las publicaciones sobre transiciones de energía y creación de nuevos mercados energéticos utilizan teorías existentes para analizar casos empíricos de transiciones. Por ejemplo, Hultman et al. (2012) exploran cómo existen factores no económicos que influyen en el cambio tecnológico e identifica cómo el sector de la energía con bajas emisiones de carbono (bioenergía y energía nuclear) sufrieron cambios significativos en los últimos 
50 años y cómo se construyeron sus mercados en tres países: Brasil, Suecia y Estados Unidos (Hultman et al., 2012); en cambio, Foxon (2013) explora escenarios de transición donde emplea un concepto de espacio de acción para explorar las interacciones dinámicas entre mercado, el gobierno y la sociedad civil. Otros estudios proponen nuevas teorías de transiciones como el de Bashmakov (2007), el cual propone que las innovaciones en el sector energético se originan para mejorar la calidad relación costo/ingreso estable más una creciente productividad energética, mientras que Geels (2002) señala que la transición entre sistemas se da por una "teoría apreciativa", donde las funciones sociales y la cultura son parte básica de los cambios tecnológicos a largo plazo en la forma en que la energía cumple con una función social.

Al buscar literatura relevante, nuestro objetivo fue identificar publicaciones clave que alberguen el debate sobre las transiciones energéticas (política de investigación, política energética, investigación energética y ciencias sociales, cambio ambiental global, innovación ambiental y transición social, y pronóstico tecnológico y cambio social) y la construcción de nuevos mercados energéticos. En suma, las ideas de estas revisiones y propuestas existentes para integrar las teorías de transición se pueden agrupar en impulsores que permitirían el establecimiento de un mercado de bio turbosina en México teniendo como línea de base todos los promotores que se mencionan en diferentes mapas de rutas para biocombustibles nacionales e internacionales (TNO, 2019; Cherp et al., 2018; ; Geels et al., 2018; Jenkins et al., 2018; IATA, 2013; Sener, 2017). En resumen, las fuerzas motoras (impulsores) que permitirán que se cree un mercado de Bio Jet en México son:

a) Políticas: el enfoque central de la perspectiva política sobre las transiciones energéticas nacionales es el cambio en las políticas que afectan los sistemas energéticos. Debido a que la mayoría de las políticas energéticas son adoptadas e implementadas por gobiernos que actúan en nombre de los estados nacionales, el estado es la principal unidad de análisis en la perspectiva política. En este sentido, la perspectiva política es diferente de las perspectivas tecnoeconómica y sociotécnica, donde los estados pueden ser actores económicos ordinarios, elementos de una red fluida, factores externos de paisaje o receptores de recomendaciones normativas, pero no el foco principal de análisis.

b) Ambientales: políticas para abordar el cambio climático mediante la reducción de gases de efecto invernadero pueden hacerse más efectivas si se logra difundir de manera clara las mejores tecnologías de baja huella de carbono.

c) Recursos naturales y su cuidado: muchas de las controversias que rodean a los sistemas energéticos es cómo estructurar políticas de cambio climático y adaptación, incluidas en las políticas financieras y medir su efecto y cómo avanza la medida contra las diferentes incertidumbres como la pérdida de fauna y vegetación, desertificación, el recurso agua, entre otros.

c) Materia prima (insumos): la biomasa tiene un papel destacado en la transición hacia la sostenibilidad, preferiblemente donde las alternativas sostenibles no están disponibles o sólo en un grado limitado; además, el uso de biomasa ofrece la posibilidad de una emisión negativa de gases de efecto invernadero.

\section{3. Barreras a la entrada}

Las restricciones de la biomasa para que se convierta en una fuente de energía alternativa, sostenible y eficiente, al menos en relación con los mercados energéticos tradicionales, se presentan a continuación.

\section{3. 1. Energética}

Para ser calificado como sostenible, el uso de una fuente de energía debe ser técnicamente factible, económicamente accesible y ambiental y socialmente viable considerando a la sociedad como un todo. Sobre todo, debe satisfacer 
las necesidades energéticas en su transición de la sociedad en su conjunto. Un tema fundamental que se pasa por alto en la narrativa general de biocombustibles, es la referencia a la tasa de retorno energético de la inversión (denominado EROI por sus siglas en inglés), que en el caso de los biocombustibles alcanza niveles más bajos que el EROI de los combustibles fósiles que es de 20-30 o más y tiene una densidad de potencia $\left(\mathrm{W} / \mathrm{m}^{2}\right)$ mucho más alta que un biocombustible, como el que se produce en Brasil base caña de azúcar. Cuando se utilizan enfoques de transición o de igualar la entrega de energía por unidad de entrada, queda claro que la biomasa no representa aún un portador de energía capaz de cumplir con las demandas de energía actual (cuadro 1).

\section{CUADRO 1}

Densidad de potencia de diferentes fuentes energéticas (Smil, 2010)

\begin{tabular}{|c|c|c|c|}
\hline \multicolumn{4}{|c|}{ Densidad de potencia [1] [DP] (W/m2) } \\
\hline \multirow[t]{3}{*}{ Fuente energética } & \multirow[t]{3}{*}{ Bajo (L) } & \multirow[t]{3}{*}{ Alto $(\mathrm{H})$} & Densidad de potencia respecto al gas natural \\
\hline & & & $D P_{\text {gas natural }}(L \& H)$ \\
\hline & & & {$\left[D P_{\text {otra fuente energética }}(H)\right]$} \\
\hline Gas natural & 200 & 2000 & * \\
\hline Carbón & 100 & 1000 & $0.2-2$ \\
\hline Solar (PV/Térmica) & 4 & 9 & $20-200$ \\
\hline Eólica & 0.5 & 1.5 & $130-1300$ \\
\hline Biomasa & 0.5 & 0.6 & $330-3000$ \\
\hline
\end{tabular}

Fuente: Smil (2010)

Nota: [1] La densidad de potencia [DP] se refiere a la tasa de liberación de energía por unidad de volumen o peso (típicamente W/ $\mathrm{m}^{2}$ o W/kg); se empleó esta unidad para poder comparar diversas fuentes alternas de energía.

\section{4. Economía}

De acuerdo con diversas fuentes, se constata que con la tecnología actual y sus costos de producción asociados, biocombustibles como el etanol y el biodiesel están muy lejos de competir con la gasolina y el diésel base petróleo Gran parte de la materia prima utilizada para la elaboración de los biocombustibles se compra en el extranjero, por lo que se está sustituyendo esta dependencia; en el caso de México, de los petrolíferos.

No se ha establecido una relación biunívoca entre los precios de venta de los biocombustibles y el precio del petróleo, debido a que en un periodo menor de un año, el petróleo bajo de precio, lo que abarató en los mercados internacionales la gasolina y diésel, mientras que los costos de venta del biodiesel y el etanol se han mantenido o aumentando ligeramente.

Referente a la experiencia de producción en Estados Unidos, el precio de la bioturbosina en promedio fue en 2013 de 7.72 USD por litro, mientras que el precio comercial de la turbosina base petróleo para ese mismo año fue de 0.75 USD. El costo de introducción fue alto, pero incluyó el desarrollo de tecnologías y fuentes de materia prima (IATA, 2013).

\section{5. Ambiental}

Respecto a las proyecciones realizadas en el proyecto Y.61023 del Instituto Mexicano del Petróleo se tiene que con las tecnologías actuales de producción se reduciría un 13\% de emisiones bajo un escenario tendencial 
a 2030. Pero estas reducciones serían mayores con la incorporación de nuevas tecnologías y materias primas (Mar, 2019).

La construcción del marco se realiza considerando que el incremento del combustible para turbo jet continua su inercia y crece a un $2.09 \%$, además de que el precio del combustible se mantiene constante en 10.32 pesos/litro.

Definidas cómo cada una de estas fuerzas puede o no apoyar la creación de un mercado de Bio Jet. Se realiza una búsqueda de las tecnologías y materias primas para la producción del Bio Jet, se diferencian acciones estratégicas y habilitadoras y se elabora el cuestionario con el que trabajarán el grupo de expertos.

En cada rubro marcarán cuando se comercialice cada combustible en el mercado nacional y de las acciones cuál sería el tema o hito por superar y cuándo. Teniendo los cuestionarios (60 expertos relacionados con el sector energéticos y 30 de otros sectores) y la literatura nacional e internacional, se construyen los mapas.

\section{5. 1. Año base para el transporte aéreo nacional.}

El objetivo primario de la industria aérea es reducir sus emisiones y para ello plantea una serie de medidas que permiten la reducción de sus impactos ambientales. De este modo, los miembros de IATA se han comprometido con los siguientes objetivos:

a) Mejorar la eficiencia del combustible en un 1.5\%/año;

b) Hacer que todo el crecimiento de la industria de la aviación sea neutral en carbono para 2020

c) Reducir las emisiones netas de $\mathrm{CO}_{2}$-eq en un 50\% para 2050, en comparación con los niveles de 2005 (gráfica 2).

De acuerdo con lo anterior, la introducción o uso extendido del JFR o Bio Jet dentro del sector aéreo es una medida que permite reducir las emisiones de $\mathrm{CO}_{2}$ debido a que los combustibles tipo Bio Jet se producen con biomasa y esta última puede considerarse como una forma de energía solar almacenada, ya que las plantas utilizan esta energía para capturar $\mathrm{CO}_{2}$ y agua a través de la fotosíntesis. Este Bio Jet es un combustible no fósil, neutro desde el punto de vista del ciclo del carbono (ciclo natural del carbono entre la tierra y el aire). Es decir, las emisiones de $\mathrm{CO}_{2}$ que se producen, al proceder de un carbono retirado de la atmósfera en el mismo ciclo biológico, no alteran el equilibrio de la concentración de carbono atmosférico y, por tanto, no incrementan el efecto invernadero; se considera que su uso contribuye a reducir las emisiones de $\mathrm{CO}_{2}$ a la atmósfera siempre que sustituya a un combustible fósil.

Teniendo como premisa este punto, se presenta la construcción de un mapa de ruta que contesta el cómo y el cuándo pasaría esto en México. Pero, primero es necesario ubicar el país en un nivel tecnológico, de mercado y de suministro de materias primas para contestar el dónde estamos. Definido el punto de partida, el método de construcción señala visualizar un objetivo y plantear una serie de acciones que nos permita llegar a él; en este caso, la introducción del Bio Jet en el mercado nacional para reducir la huella de carbono del sector aéreo, identificando también posibles barreras o riesgo.

\section{5. 2. Mapa de ruta}

El desarrollo de un mapa de ruta es proporcionar una visión general y proyecciones de puntos estratégicos o hitos que conducen al desarrollo y despliegue exitoso de un objetivo específico; en este caso, el uso extendido de combustibles alternativos para aviones Bio Jet. Este mapa de ruta se basa en la publicación del Mapa de Ruta Tecnológica Bioturbosina (Sener, 2017), en pláticas con expertos en el tema durante 2016-2017 y en información pública disponible. El compendio de estos elementos se aproxima a un consenso de diversos puntos de 
vista que se pudiera considerar un proxi de posibles actividades y necesidades para la inclusión y desarrollo de un mercado nacional de Bio Jet en el próximo medio siglo.

a) Mapa de ruta de nivel 1

Se presenta una descripción general de los impulsores que generan una transición a combustibles alternativos para aviones (es decir, un precio alto de petróleo, cambio climático, reducción de producción de crudo) (figura 6).

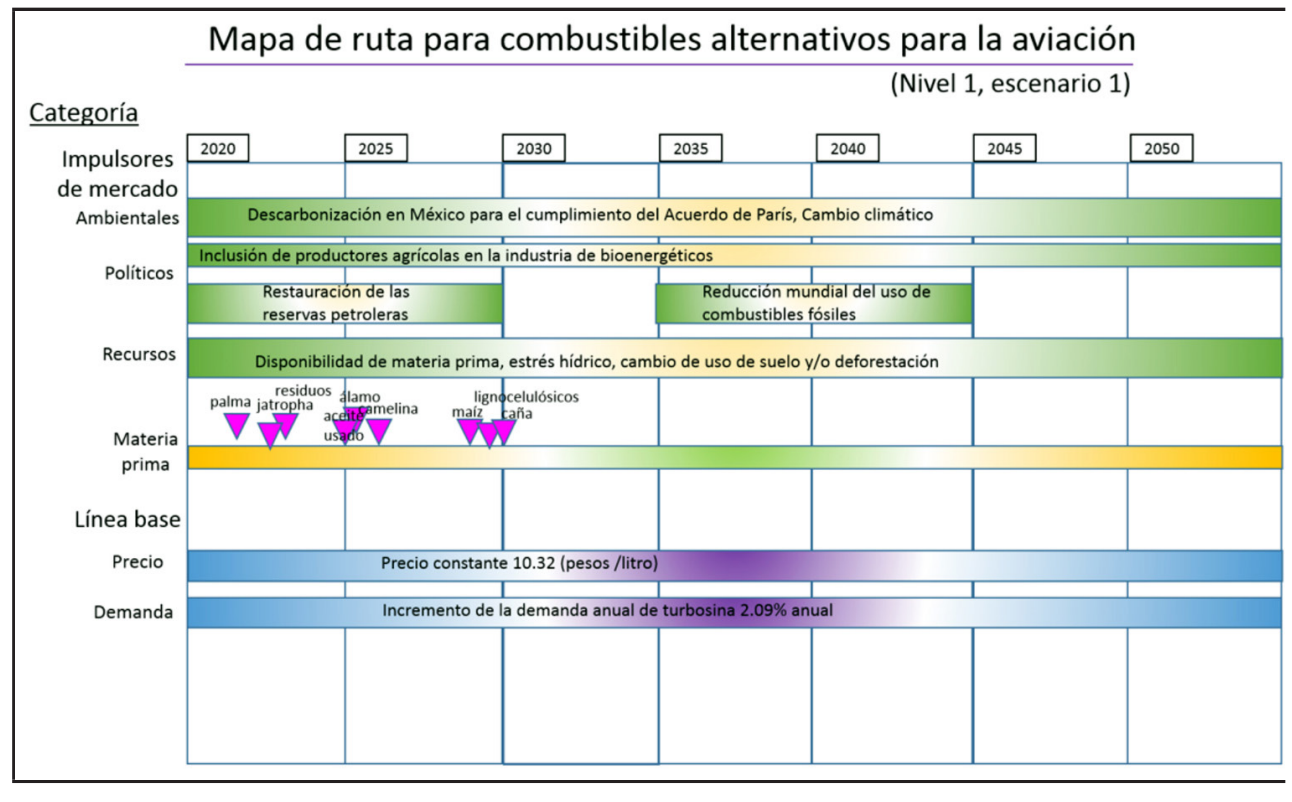

FIGURA 6

Mapa de ruta para la producción de Bio Jet en México/nivel 1

Fuente: elaboración propia.

b) Mapa de ruta de nivel 2

En esta hoja o mapa de ruta se identifican cuatro áreas clave en las que se deben realizarse diversas actividades para alcanzar un uso extendido de combustibles alternativos en la aviación como son:

- Acciones estratégicas para el despliegue y fortalecimiento de una cadena de valor que amplié un mercado de Bio Jet nacional (figura 5)

-Economía/negocios: identificar, planificar y coordinar pasos clave en el desarrollo de una industria de combustibles alternativos respecto a la adquisición, oferta y demanda para reducir riesgos de inversión, ambientales y sociales.

-Participación sistemática gobierno/industria en todas las fases de desarrollo de producto, entre diversas cadenas de valor industrial.

- Cadena de valor que contribuya a la excelencia global, nuevo conocimiento y nuevas formas de participación.

- Acciones habilitadoras que se identifican como aquellos campos del conocimiento que son inductores de innovaciones en diversos sectores como son el medioambiente, la economía local y el desarrollo social y científico. Estas acciones habilitadoras tienden a potenciar y detonar una disrupción en los mercados energéticos actuales y modificarlos en los próximos 10 a 15 años. Las acciones habilitadoras se ubican en los siguientes campos (figura 7 y figura 8) 
-I\&DT: las tecnologías nuevas que un inicio no pueden competir dentro de los regímenes dominantes (por ejemplo, porque son demasiado costosas o complicadas) surgen en nichos protegidos, donde pueden madurar y volverse competitivos, de aquí que el apoyo por la ciencia como parte de las actividades gubernamentales fundamental. El enfoque de gestión de nicho estratégico (SNM) en particular enfatiza la necesidad de fomentar nichos innovadores para facilitar la innovación tecnológica (René et al., 1998). En este punto se identifica, planifica y coordina actividades necesarias para la superación de barreras técnicas que permitan el desarrollo y producción de combustibles alternativos.

-Sociales: uno de los marcos más influyentes en el análisis de transición social (Gelles, 2012) señala que debido a la resistencia del régimen, los nichos no desplazan automáticamente a los regímenes establecidos, incluso cuando se vuelven más efectivos en el cumplimiento de una función social relevante. Para que un nicho reemplace a un régimen establecido, el régimen primero debe ser desestabilizado; por ejemplo, por presiones externas (en este caso por cuestiones de cambio climático). La desestabilización del sistema original puede ocurrir a lo largo de distintas vías, las cuales representan un cambio rápido no lineal, además de demostrar e incluir las bondades de la transición energética. Por ejemplo, estudios recientes indican que la bioenergía tiene un mayor impacto positivo en la creación de empleo en las zonas rurales que otras fuentes de energía (Edenhofer et al., 2011). Se reporta que las industrias de biocombustibles emplean aproximadamente 100 veces más trabajadores por unidad de energía producida que la industria de combustibles fósiles (Kojima y Johnson, 2005). Así, el desarrollo de una industria bioenergética busca la creación de empleo altamente cualificado, que mejore la calidad de vida y cree prosperidad futura; se tienen como objetivos primordiales la inclusión de las actividades agrícolas en la cadena de valor del Bio Jet y lograr la conectividad entre sistemas energéticos/mercados/individuos.

-Medioambiente: el enfoque central de la perspectiva ambiental sobre las transiciones energéticas es el cambio a un desarrollo de bajo carbono que afecta a los sistemas energéticos actuales. Este cambio se estudia dentro de varios dominios de la ciencia desde el estrés hídrico hasta la deforestación y pérdida de biota por cambios en el sistema por un uso extendido de bioenergéticos para generar recomendaciones normativas. Sin embargo, el foco principal del análisis es la evaluación integral de los impactos ambientales de los biocombustibles y de los tipo drop-in ${ }^{1}$ a mediano y corto plazo y su contribución a la reducción de emisiones.

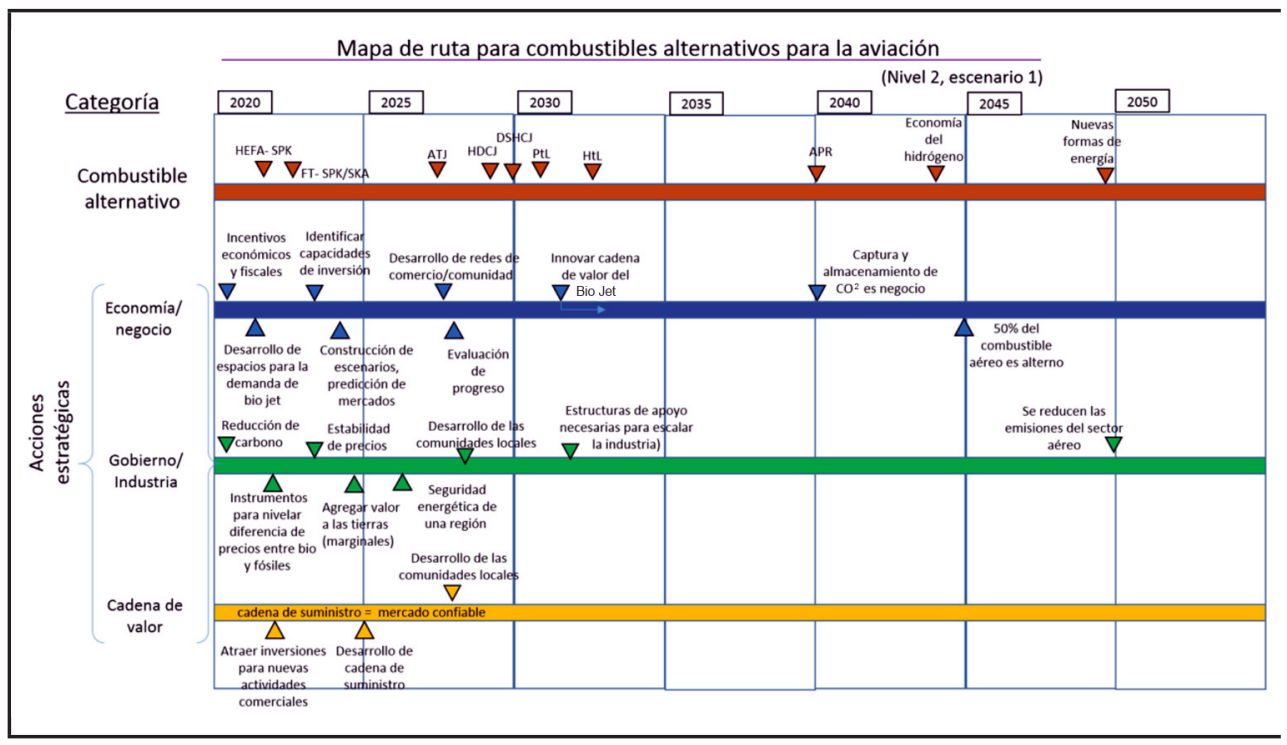

FIGURA 7

Mapa de ruta para la producción de Bio Jet en México/nivel 2 (acciones estratégicas) Fuente: elaboración propia. 


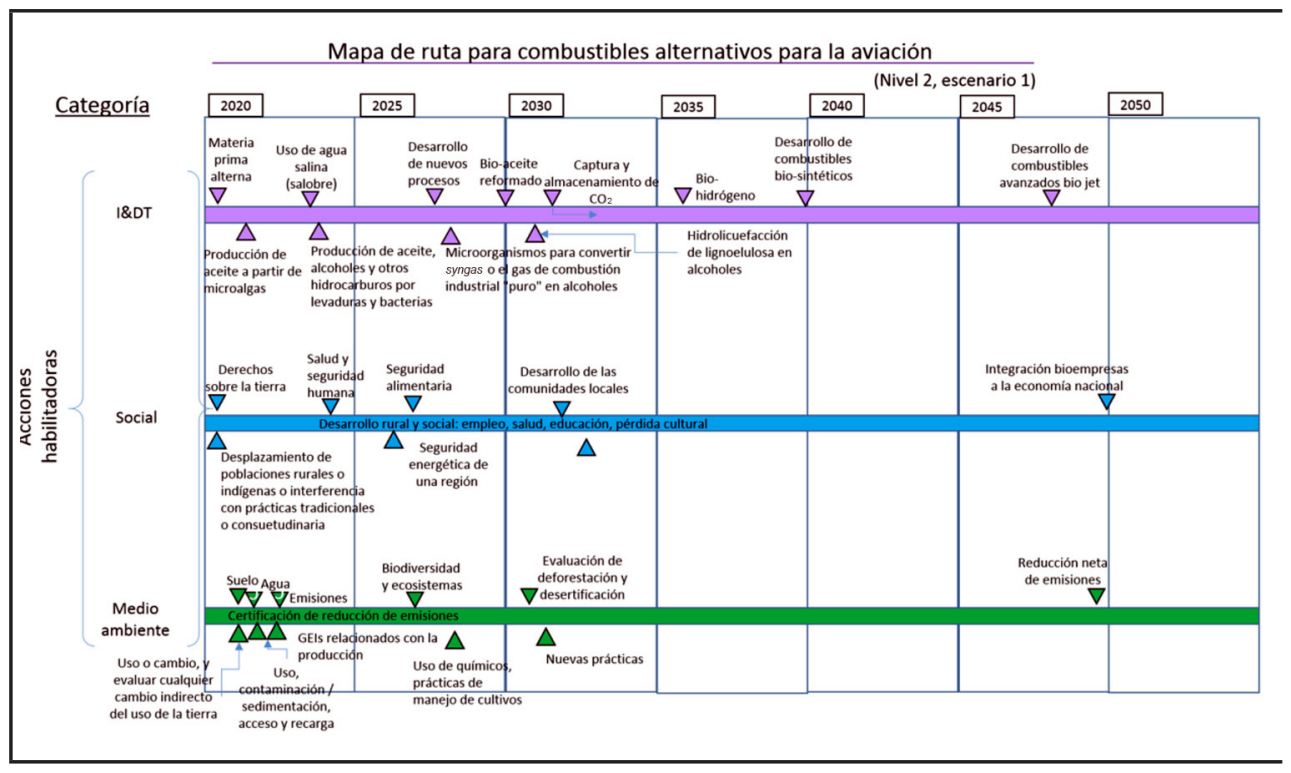

FIGURA 8

Mapa de ruta para la producción de Bio Jet en México/ nivel 2 (acciones habilitadoras) Fuente: elaboración propia.

\section{Resultados del mapa de ruta}

Este análisis sobre las acciones que permitirán que entren y se desarrolle un mercado de Bio Jet en México permitió observar los siguientes resultados:

a) Identificar los estudios necesarios sobre combustibles alternativos claves a corto plazo con respecto a propiedades, especificaciones, producción, procesamiento y uso: por ejemplo el mapa de ruta inicia con el empleo de un proceso de ésteres y ácidos grasos hidroprocesados (HEFA o HEFA-SPK) que, de acuerdo con la literatura al realizar un análisis de ciclo de vida y empleando diversas materias primas que van desde aceite de palma, jatropha, camelina, residuos, entre otros, su huella es $58 \%$ menor en promedio que la de la turbosina que en gran medida dependerá de la materia prima de base. Respecto a la síntesis FT, la producción de hidrógeno para el proceso es el punto por analizar si bien se considera una ruta donde se puede usar electricidad renovable para convertir el hidrógeno requerido en el proceso de hidrólisis (el cual se denomina StL o PtL). Este proceso denominado PtL (Power to liquids, es decir, generación de electricidad a combustibles líquidos) es una ruta de producción que se calcula tenga costos variables de producción altos aunque disminuya el costo de generación con renovable en el corto plazo (Searle y Christensen, 2018). El análisis técnico-económico de PtL sugiere que los gastos de capital y los costos de electricidad de entrada pueden ser altos; se calcula un rango de costos de 2.8 USD a 7.8 USD por litro de capacidad anual dependiendo de la configuración que se emplee (Schmidt et al., 2016; Mar, 2019) (figura 9). El siguiente proceso en el mapa es Alcohol to Jet (ATJ-SPK), en el cual se sintetiza el alcohol para producir queroseno y de ahí Bio Jet. Esta ruta de conversión utiliza la fermentación para convertir azúcares, almidones o celulosa hidrolizada en un alcohol intermedio; las emisiones que se tiene por este proceso todavía son $43 \%$ en promedio más altas en comparación con otras tecnologías de reducción de emisiones, ya que la obtención de alcohol varía de materia prima que puede ser alcohol de caña, maíz, de rastrojo de maíz, etc. Y cada uno de éstos presenta una huella por materia prima que difiere más que del proceso mismo. Entonces, no resulta recomendable apostar en el corto y mediano plazo por estas tecnologías, sino que se sugiere la realización de estudios que avalen estas rutas.

b) Los requerimientos de datos para formar la base del proceso para garantizar la reducción de impactos ambientales por el uso de Bio Jet: 
Si la meta de las asociaciones que se están creando en el país tienen como objetivo el uso de biocombustibles producidos de forma sostenible para reducir las emisiones de dióxido de carbono $\left(\mathrm{CO}_{2}\right)$ entre $50 \%$ y $80 \%$ respecto a los combustibles derivados del petróleo convencional, es importante avalar esta información con las materias propuestas como jatropha y camelina que al no ser cultivos endémicos tal vez su consumo de agua y el uso de agroquímicos para alcanzar los niveles de producción necesarios para lograr un mercado podría afectar negativamente en recursos como tierra y agua teniendo como resultado final una huella de carbono más alta que otras materias primas o que el mismo petróleo.

c) Identificación de áreas de oportunidad en investigación y financiamiento clave para el desarrollo de la cadena de valor con base en los diferentes estudios realizados para el país (Mar, 2019).

d) Puntos de medición/cambio/adaptación del progreso en el desarrollo y despliegue de combustibles alternativos cuando se actualiza el mapa de ruta, que a su vez permite evaluar avances para el uso extendido de combustibles alternativos.

e) Mecanismo de interacción con los diferentes habilitadores en puntos clave para apoyar/desarrollar/desplegar un mercado de bioenergéticos nacional, así como puntos de evaluación ambiental para definir nuevas acciones a emprender.

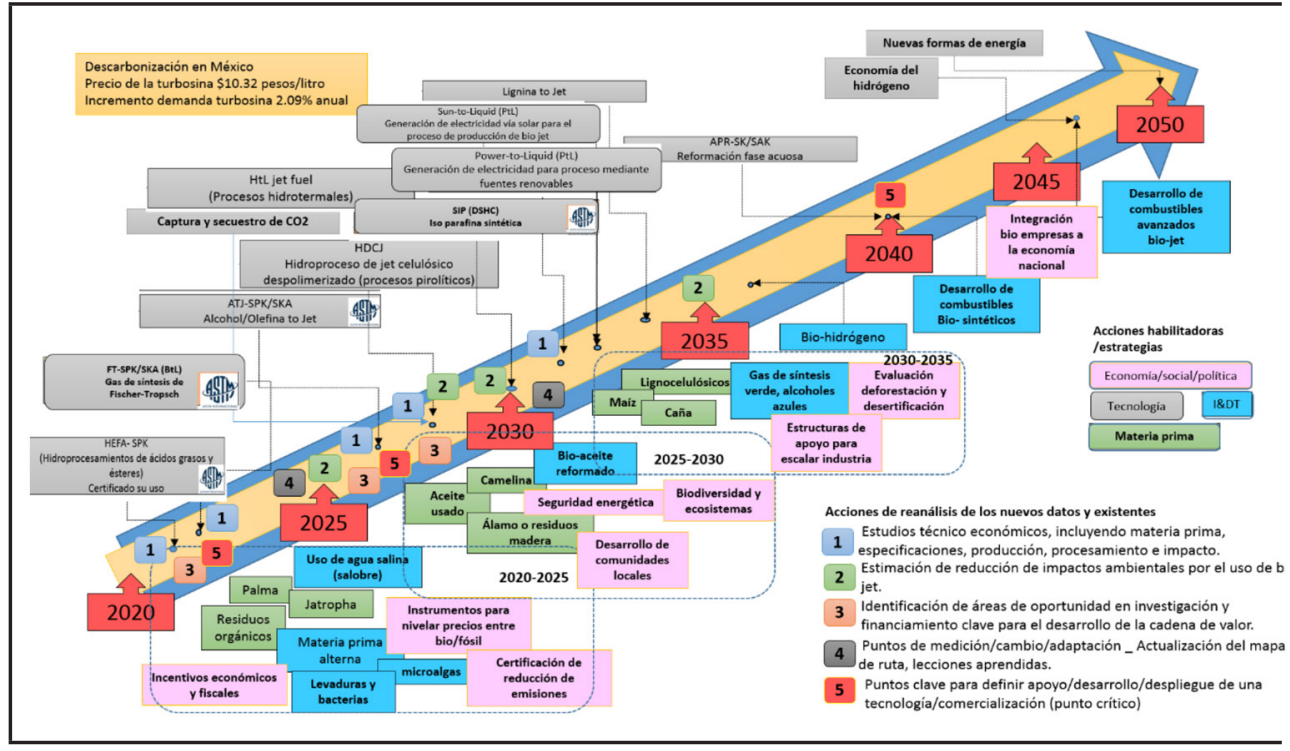

FIGURA 9

Mapa de ruta para la construcción de un mercado de Bio Jet nacional Fuente: elaboración propia.

\section{Conclusiones}

En temas relacionados con el uso de bioenergéticos en países emergentes, visualizar y promover un futuro sostenible es una tarea complicada. La consecuencia negativa de planear sin límites claros de conciencia social y realidad científica es la posibilidad de promover un desarrollo socioeconómico no sostenible por tener metas desalineadas y no alcanzar hitos por una ejecución pobre o sin posibilidades de éxito de las acciones propuestas. Esto marca retos que requieren tomarse en cuenta para que cualquier planeación se implante de manera adecuada y tener impactos positivos en términos de atender cuestiones tanto de cambio climático como de la pobreza en el campo.

A pesar de estos riesgos, la incorporación del concepto bio es un punto clave de la respuesta a los desafíos de la sostenibilidad. En este caso, el transporte aéreo desesperadamente requiere metas y objetivos que incluyan bioenergéticos y así atender algunas de sus necesidades de sostenibilidad futura. Con esto en mente, se desarrolló 
un mapa de ruta de incorporación de bioenergéticos a la matriz de combustibles del sector de transporte aéreo a través de metodologías de inteligencia tecnológica.

Para ser una alternativa viable para el petróleo, un biocombustible debería proporcionar una ganancia neta de energía, ofrecer claros beneficios ambientales y económicos y no reducir el suministro de alimentos o aumentar sus costos. Los biocombustibles no cumplen aún con estos requisitos; por ello, es importante tener presente estos objetivos en el desarrollo del mapa de ruta para llegar a estas metas e introducir los biocombustibles en la matriz energética nacional. El costo se presenta como un obstáculo importante para la comercialización de biocombustibles en comparación con los combustibles derivados del petróleo. La menor densidad de energía y el precio de las materias primas encarecen los biocombustibles al producir calor. Y cuanto mayor sea el contenido de biocombustible del combustible, menor será la densidad de energía y, por lo tanto, la eficiencia energética.

El mapa resultante es una herramienta de análisis para la planeación energética desde el punto de gobierno de relevancia. Sin embargo, se pudo observar que algunos elementos de análisis eran maleables y que el mapa contenía puntos debatibles y discordantes con la realidad socioeconómica y científica de nuestra economía emergente. Así, con el objetivo de eliminar estos cuellos de botella con consecuencias negativas para el futuro, el mapa se modificó al identificar puntos de inflexión que podían afectar la puesta en marcha de un mercado de Bio Jet-fuel nacional y replantear las necesidades de I\&DT enfocando el mapa a que impulse una verdadera cadena de valor nacional respecto a los bioenergéticos.

\section{ReFERENCIAS}

ASA (Aeropuertos y Servicios Auxiliares).(2018).ASA. Disponible en https://datos.gob.mx/busca/organization/asa Bashmakov, I. (2007). Three laws of energy transitions. Energy Policy, 35(7), 3583-3594. https://doi.or$\mathrm{g} / 10.1016 / \mathrm{j}$. enpol.2006.12.023

Borghesi, S., \& Montini, M. (2016). The Best (and Worst) of GHG Emission Trading Systems: Comparing the EU ETS with its Followers. Frontiers in Energy Research, 4, 27.

Cherp, A. Vinichenko, V., Jewell, J., Brutschin, E., \& Sovacool, B. K. (2018). Integrating techno-economic, socio-technical and political perspectives on national energy transitions: a meta-theoretical framework. Energy Research \& Social Science, 37, 175-190.

Edenhofer, O., Pichs-Madruga, R., Sokona, Y., \& Seyboth K. (2011). Bioenergy. In IPCC Special Report on Renewable Energy Sources and Climate Change Mitigation. Cambridge and New York. Cambridge University Press. Retrieved from https://www.researchgate.net/publication/281688088

Foxon, T. (2013). Transition pathways for a UK low carbon electricity future. Energy Policy, 10-24.

Geels, F. (2002). Technological transitions as evolutionary reconfiguration processes: A multi-level perspective and a case-study. Research Policy, 31, 8-9, 1257-1274. https://doi.org/10.1016/S0048-7333(02)00062-8

Geels, F., Sorrell S., Schwanen, T., Jenkins, J., \& Sovacool, B. K. (2018). Reducing demand through low-energy innovation: A sociotechnical review and critical research agenda. Energy Research \& Social Science, 40, 23-35

Hall, D., Pavlenko, N., \& Lutsey, N. (2018). Beyond road vehicles: Survey of zero-emission technology options across the transport sector. International council on clean transportation. Retrieved from https://theicct. org/sites/default/files/publications/Beyond_Road_ZEV_Working_Paper_20180718.pdf

Hultman, N., Malone, E., Runci, P. Carlock, G., \& Anderson, K. (2012). AndersonFactors in low-carbon energy transformations: comparing nuclear and bioenergy in Brazil, Sweden, and the United States. Energy Policy, 131-146. 
IATA (International Air Transport Association) (2009). Annual Report. Retrieved from https://www.iata.org/ contentassets/c81222d96c9a4e0bb4ff6ced0126f0bb/iataannualreport2009.pdf

IATA (International Air Transport Association) (2013). Technoloy Roadmap 4th edition. Retrieved from https:// www.iata.org/whatwedo/environment/Documents/technology-roadmap-2013.pdf

IATA (International Air Transport Association). (2017).20-Year Air Passenger Forecast. Retrieved from https:// www.iata.org/pressroom/pr/Documents/2017-10-24-01-sp.pdf

IPCC (Intergovernmental Panel on Climate Change). (2018). Global Warming of $1.5^{\circ} \mathrm{C}$, an IPCC special report on the impacts of global warming of $1.5^{\circ} \mathrm{C}$ above pre-industrial levels and related global greenhouse gas emission pathways, in the context of strengthening the global response to the threat of climate change, sustainable development, and efforts to eradicate poverty. Retrieved from https://report.ipcc.ch/sr15/pdf/sr15_spm_final.pdf

Kojima, M., \& Johnson. T (2005). Potential for biofuels for transport in developing countries. World Bank. Retrieved from http://documents.worldbank.org/curated/en/939911468150318214/pdf/ESM3120PAPER0Biofuels.pdf

LPDB (Ley de Promoción y Desarrollo de los Bioenergéticos). (2008). Diario Oficial de la Federación.

Jenkins, K., Sovacoolb, B., \& McCauleyd, D. (2018). Humanizing sociotechnical transitions through energy justice: An ethical framework for global transformative change. Energy Policy, 117, 66-74. https://doi. org/10.1016/j.enpol.2018.02.036

Mar, E. (2019). Informe técnico Proyecto Y.61023 “Clúster Bioturbosina”. La economía de producir combustible de aviación sostenible: Caso México. Instituto Mexicano del Petróleo.

Nielsen-Gammon, J. (2007). "An Inconvenient Truth": The scientific argument. GeoJournal, 70(1), 21-26.

René, K., Schot, J., \& Hoogma, R. (1998). Regime shifts to sustainability through processes of niche formation: The approach of strategic niche management. Journal Technology Analysis \& Strategic Management, 10, 2.

Schmidt, P., Weindorf, W., Roth, A., Batteiger, V., \& Riegel, F. (2016). Power-to-liquids: Potentials andperspectivesfor the future supply of renewable aviation fuel. German Environment Agency. Retrieved from www.umweltbundesamt.de/sites/default/files/medien/377/publikationen/161005_uba_hintergrund_ptl_barrierrefrei.pdf

Searle, S., \& Christensen, A. (2018). Decarbonization potential of electrofuels in the European Union. International Council on Clean Transportation. Retrieved from www.theicct.org/sites/default/files/publications/ Electrofuels_Decarbonization_EU_20180920.pdf

Sener (2017). Mapa de ruta tecnológica bioturbosina. Secretaría de Energía (Sener). Disponible en https://www. gob.mx/cms/uploads/attachment/file/324219/MRT_Bioturbosina_Final.pdf

Smil, V. (2010). Energy: Myths and realities. Washington: The AEI Press.

Thunberg, G. (2019). Our house is on fire. World Economic Forum, 2019. Retrieved from https://www.weforum. org/agenda/2019/01/our-house-is-on-fire-16-year-old-greta-thunberg-speaks-truth-to-power/

TNO (Netherlands Organisation for Applied Scientific Research). (2019). Towards broad support for the energy transition.TNO. Retrieved from https://www.tno.nl/en/focus-areas/ecn-part-of-tno/roadmaps/towardsbroad-support-for-the-energy-transition/

\section{Notas}

[1] Los biocombustibles drop-in o "gota a gota" se definen como "biohidrocarburos líquidos que son funcionalmente equivalentes a los combustibles base petróleo y son totalmente compatibles con la infraestructura petrolera existente. Lo que no ocurre con el etanol vs gasolina o diésel vs biodiésel.

CC BY-NC-ND 